\title{
Dual $Q$-switched laser outputs from a single lasing medium using an intracavity MEMS micromirror array
}

\author{
Ralf Bauer, ${ }^{*}$ Walter Lubeigt, and Deepak Uttamchandani \\ Centre for Microsystems and Photonics, Department of Electronic and Electrical Engineering, \\ University of Strathclyde, Glasgow G1 $1 X W, U K$ \\ *Corresponding author: ralf.bauer@strath.ac.uk
}

Received June 15, 2012; revised July 18, 2012; accepted July 22, 2012;

posted July 23, 2012 (Doc. ID 170706); published August 23, 2012

\begin{abstract}
An intracavity array of individually controlled microelectromechanical system scanning micromirrors was used to actively $Q$-switch a single side-pumped Nd:YAG gain medium. Two equal power independent laser outputs were simultaneously obtained by separate actuation of two adjacent micromirrors with a combined average output power of $125 \mathrm{~mW}$. Pulse durations of $28 \mathrm{~ns}$ FWHM at $8.7 \mathrm{kHz}$ repetition frequency and $34 \mathrm{~ns}$ FWHM at $7.9 \mathrm{kHz}$ repetition frequency were observed for the two output beams with beam quality factors $M^{2}$ of 1.2 and 1.1 and peak powers of $253 \mathrm{~W}$ and $232 \mathrm{~W}$, respectively. (c) 2012 Optical Society of America

OCIS codes: $140.3290,140.3540,140.3580,230.4685$.
\end{abstract}

The use of microelectromechanical systems (MEMS) as active elements in laser systems has been the subject of increasing interest in recent years due to dimensional compatibility with laser beams, low power consumption, low-cost high-volume fabrication and ease of integration with laser gain media, thereby presenting the prospect for highly integrated, compact laser systems. These advantages have been exploited in wavelength tuning of microchip lasers [1,2] and fiber lasers [3] where the MEMS devices have been used as both intracavity and extracavity elements. In addition, MEMS scanning micromirrors appear as viable alternatives to acousto- or electro-optic modulators for $Q$-switched or mode-locked laser sources. Driving the micromirrors at their mechanical resonance allows high speed modulation of the intracavity optical field due to the combination of small feature sizes (mirror diameter usually $<1 \mathrm{~mm}$ ) and high structural stiffness, leading to high scanning speeds and, ultimately, fast changes of the cavity quality. Cantilever-type MEMS have been used in fiber lasers to achieve pulse durations ranging from tens of nanoseconds [4] to a few picoseconds [5] with active and hybrid $Q$-switch designs, respectively. By using membrane deformable mirrors, $Q$-switched [므] ] and mode-locked []] operations have also been demonstrated in fiber lasers. Resonantly oscillating piezoelectric [9] and single-crystal silicon mirrors [10] have generated active $Q$-switch actuation in microchip and side-pumped Nd-rod lasers, respectively. The compact footprint of the MEMS devices allows integration schemes not possible with traditional approaches using larger laser optics, thereby opening new concepts and avenues for the design of solid-state lasers.

Building upon our previous work on actively $Q$ switched solid-state lasers using MEMS [10] we present in this Letter the novel use of an array of electrostatic resonantly actuated MEMS micromirrors as active $Q$ switch elements to generate a pair of individually controlled laser beams from a single gain medium, where the laser outputs are separated both spatially and in their temporal characteristics. To achieve this, the combdrive-actuated micromirrors are driven to oscillate at their torsional resonance mode to achieve fast angular scanning and therefore rapid $Q$ changes of the laser cavity. Multiple laser output beams have previously been shown by a $4 \times 4$ VCSEL array with total peak output power of up to $123 \mathrm{~W}$ by short pulsed injection [11]. The implementation of MEMS arrays as active $Q$ switches in Nd-based laser cavities demonstrated in this Letter enable the prospect for high pulse energies. Consequently, this individual beam control concept will have significant applications in defence, industrial, or imaging sectors.

The layout and scanning electron microscopy (SEM) image of the $2 \times 2$ micromirror array used for this work are shown in Figs. 1(a) and 1(b). Each mirror has a $700 \mu \mathrm{m}$ diameter reflective surface connected by $750 \mu \mathrm{m}$ long torsion springs of varying widths to generate different resonance frequencies for the movement modes. Mirrors $\mathrm{M} 1$ and $\mathrm{M} 2$, used in the work presented, have torsion spring widths of 12 and $13 \mu \mathrm{m}$, respectively. The cavity alignment and actuation of only two of the four mirrors of the array was undertaken to ensure that the area of the mirrors matched with the cross-section area of the laser

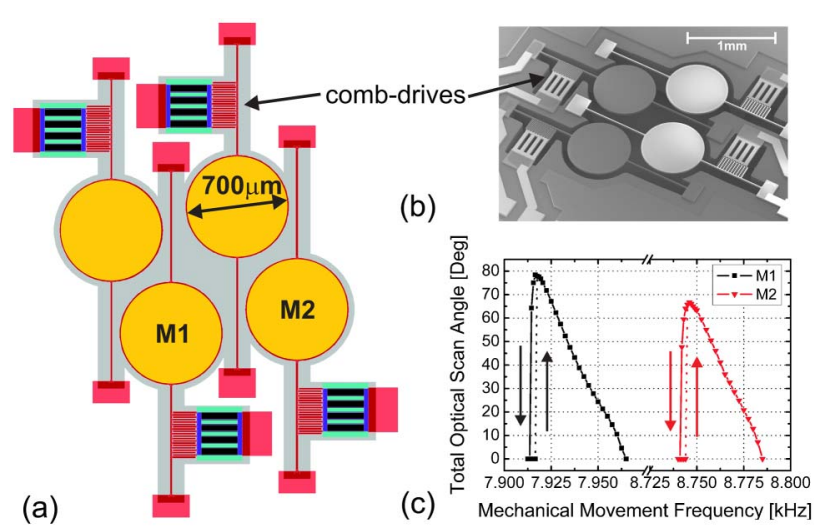

Fig. 1. (Color online) (a) Layout and (b) SEM image of fabricated array (N.B. the color difference of the micromirrors is due to charge build up during image acquisition) with (c) angular frequency response of M1 and M2. 
rod used in this work. Each mirror was actuated by a single electrostatic comb-drive actuator with 10 interdigitated fingers which are $10 \mu \mathrm{m}$ wide and have an overlap length of $150 \mu \mathrm{m}$. The devices were fabricated to our designs using a commercial multiuser silicon-on-insulator process with a $25 \mu \mathrm{m}$ thick phosphorus doped single crystal device layer, supplied by MEMSCAP Inc. [12]. A backside deep reactive ion etch step through the $400 \mu \mathrm{m}$ thick substrate fully released the mirror surfaces, actuators, and torsion springs. An in-house thermal evaporation postfabrication coating of gold with $240 \mathrm{~nm}$ thickness was applied to the surfaces of the micromirrors to increase their reflectivity $(R)$. Using a probe laser beam, the reflectivity achieved was measured to be $R=96 \%$ at $1064 \mathrm{~nm}$.

Torsional resonance movement of each micromirror was generated by the application of an AC voltage signal with a frequency close to a multiple of the micromirror mechanical resonance frequency [13]. Using a probe $\mathrm{He}-$ Ne laser, the maximum optical scan angle of both micromirrors was measured and is shown in Fig. 1(c). Both mirrors were actuated using a square-wave signal with $200 \mathrm{~V}_{p-p}$ amplitude and $100 \mathrm{~V}$ DC offset and were driven at a frequency double that of the mechanical resonance frequency. M1 exhibited a lower mechanical resonance frequency $(7.916 \mathrm{kHz})$ due to the thinner spring design and the resulting lower stiffness of the springs. This also led to a higher angular scan range with a maximum of $78^{\circ}$ total optical scan angle (TOSA) compared to that obtained for M2 $\left(67^{\circ} \mathrm{TOSA}\right)$. The mechanical resonance frequency of M2 was measured to be $8.748 \mathrm{kHz}$. In both cases, a slight hysteresis (below $0.05 \%$ in respect to the resonance frequency) was observed with higher scan angles obtained when sweeping down from a frequency higher than the resonance frequency. This hysteresis, together with the nonsymmetric shape of the frequency response curve, is due to spring softening nonlinearities, introduced by mechanical and capacitive nonlinear effects in the resonating device [14]. The mechanical resonance build up and decay times were measured and calculated to be approximately $200 \mathrm{~ms}$ to reach full angular movement, which will represent the upper limit of the rise and fall times of the $Q$-switched pulse train.

The mirrors exhibited an intrinsic surface curvature due to internal material stress in the silicon device layer during fabrication. This concave curvature increased with the deposition of the gold coating due to a mismatch in coefficients of thermal expansion between gold and silicon. The resulting curvature profiles of both micromirrors were measured using a VEECO NT1100 white light interferometer. Mirrors M1 and M2 had a radius-ofcurvature (ROC) of $-0.22 \mathrm{~m}$ and $-0.35 \mathrm{~m}$, respectively. This difference in ROC values originates from a non uniform gold deposition, which showed mask edging effects on the surface of M2. However, the parallelism of both mirrors was not impacted by this edging effect, thus still allowing simultaneous alignment of a dual mirror codirectional laser cavity. In addition, the coating discrepancy was found to have limited impact on the performance of each laser cavity.

As shown in Fig. 2, a pair of two-mirror laser cavities were built around a commercially acquired, sidepumped, Nd:YAG laser rod $(63 \mathrm{~mm}$ length and $3 \mathrm{~mm}$

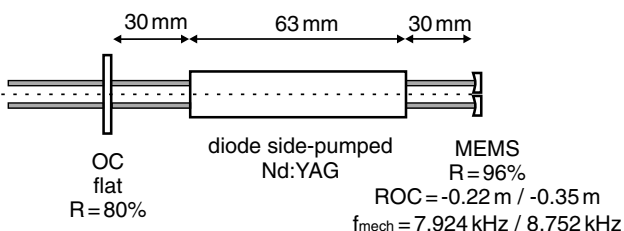

Fig. 2. Laser cavity setup for $Q$-switched dual mirror actuation of MEMS array.

diameter, doping concentration 0.6 at. \%) with a $R=$ $80 \%$ flat output coupler (OC) and the MEMS array as the end mirror. To achieve $Q$-switched output, both mirrors M1 and M2, whose centers are separated by $870 \mu \mathrm{m}$, were aligned symmetrically around the center of the rod axis. Both mirrors were individually actuated with a $200 \mathrm{~V}_{p-p}$ square-wave signal with frequencies of $f_{\mathrm{M} 1}=$ $15.848 \mathrm{kHz}$ and $f_{\mathrm{M} 2}=17.504 \mathrm{kHz}$. These frequencies were chosen so that similar scan angles for both mirrors were obtained. Driving frequencies that are slightly above the optimum driving values ensured that the resonance movement could not be annihilated by thermally induced variation of the mechanical resonance frequency. A minimal opposite orthogonal tilt $\left(\approx 0.015^{\circ}\right)$ of the mirror surface on every successive pass through the alignment was observed in addition to the scanning movement, due to the nonsymmetric actuation point of the electrostatic force.

The beam profile of both laser outputs resulting from actuation of M1 and M2 was measured using a Thorlabs BP104-IR scanning slit beam profiler and the results can be seen in Fig. 3(a) with a center-to-center separation of $\approx 1.2 \mathrm{~mm}$ at a distance of $50 \mathrm{~mm}$ from the OC. The combined average output power of both beams was measured as $P_{\text {avg }}=125 \mathrm{~mW}$ with an optical incident pump power of $100 \mathrm{~W}$ from the side-pump diode stacks. The average output power was purposely kept below $150 \mathrm{~mW}$ in order to avoid thermally induced variations of the mirror resonance and surface curvature, originating from the absorption of intracavity optical radiation in the gold coating layer of the micromirrors. Using the beam profiler, the beam quality factor $M^{2}$ for each beam was experimentally determined at the average combined output power of $125 \mathrm{~mW}$. Both outputs showed similar values for their $x$ and $y$ axes with $M_{\mathrm{M} 1}^{2}=1.1$ and $M_{\mathrm{M} 2}^{2}=1.2$. To evaluate the independent behavior of the two laser cavities, an intracavity knife-edge was used to block each beam individually. In both occasions, the characteristics
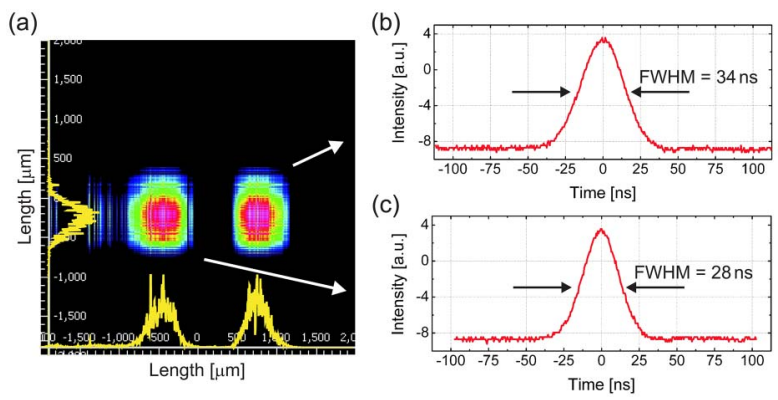

Fig. 3. (Color online) $Q$-switch output with (a) spatial beam profile $50 \mathrm{~mm}$ after the $\mathrm{OC}$ and temporal pulse shapes for output of cavity using (b) M1 and (c) M2. 


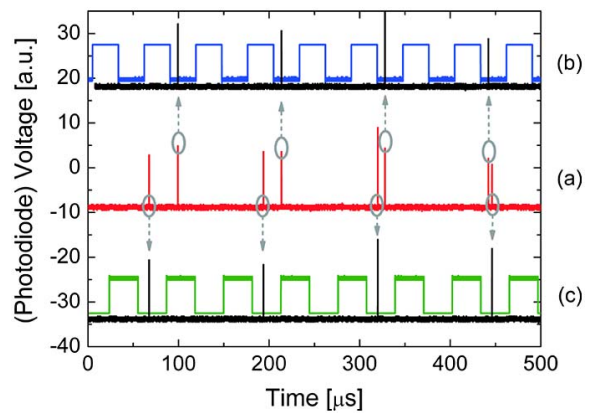

Fig. 4. (Color online) (a) Pulse train of both laser beams, laser output and driving waveform for (b) M2 and (c) M1.

of the remaining oscillating beam remained unchanged leading us to believe that no gain competition exists between the two oscillating fields.

Both pulsed output beams were focused onto a 1.5 GHz Si photodiode and the resulting pulse trains are displayed in Fig. 4(a). Both pulse trains can be distinguished by blocking one of the output beams with a knife edge with the results displayed in Figs. 4(b) and $\underline{4(c)}$ including the corresponding electrical actuation signals. The pulse repetition frequency (PRF) for M1 and M2 were measured at 7.929 and $8.752 \mathrm{kHz}$, respectively, and correspond to the mechanical movement frequency of each micromirror. Since the laser beams were located close to the edge of the mirror surface, the slight orthogonal tilt mentioned in the previous section meant that only a single pulse per mechanical movement cycle was obtained. The temporal pulse profiles are shown in Figs. 3(b) and 3(c) for the cavities featuring M1 and M2, respectively. The measured full width half-maximum (FWHM) pulse duration was 34 ns for the cavity using M1 and 28 ns for the cavity using M2. Cavity limited pulse durations were obtained over a frequency range of $\pm 20 \mathrm{~Hz}$ for $\mathrm{M} 1$ and $\pm 12 \mathrm{~Hz}$ for M2 around the driving frequencies. The average output power of both pulses was measured to be equal. Therefore, pulse energies of 7.9 and $7.1 \mu \mathrm{J}$ and pulse peak powers of 232 and $253 \mathrm{~W}$ are calculated for the laser outputs of M1 and M2, respectively.

The theoretical minimum pulse duration $\tau_{\min }$ for the presented laser cavity can be estimated using the cavity round-trip time and the unsaturated round-trip gain of the laser gain medium with [15]:

$$
\tau_{\min } \approx 8.1 \cdot \frac{\tau_{r}}{\ln G} \text {. }
$$

The cavity round-trip time was calculated as $\tau_{r}=0.82 \mathrm{~ns}$ while the unsaturated round trip gain was estimated to be $G=1.24$ since the laser operated close to the laser threshold. This leads to a theoretical minimum pulse duration of $30 \mathrm{~ns}$ for the setup presented. Both pulse durations are close to this value, with small deviations due to the nonhomogeneous gold coating of the array mirrors, with lower reflectivity of the mirrors leading to shorter possible pulse durations in accordance with (1).

In conclusion, we have demonstrated for the first time, to the best of our knowledge, individual controllable multiple $Q$-switched laser outputs generated from a common solid-state gain medium and an intracavity MEMS micromirror array. The individual controllability of each mirror of the array leads to new opportunities for laser pulse timing and beam steering using miniature, low-cost optical MEMS devices, with potential applications in defense, industrial, and imaging sectors. An increase in the mirror array density by reduction of the mirror size or by an alternative actuation scheme can lead to a higher number of laser outputs with higher PRFs due to the lower mass in the mechanical oscillation system. Finally, power scaling and increased laser efficiency will be obtained with higher reflectivity mirror coatings using dielectric materials.

The authors thank Dr. J. Mackersie for providing the gold coatings.

\section{References}

1. X. M. Zhang, A. Q. Liu, D. Y. Tang, and C. Lu, Appl. Phys. Lett. 84, 329 (2004).

2. S. Schilt, K. Zogal, B. Kögel, P. Meissner, M. Maute, R. Protasio, and M.-C. Amann, Appl. Phys. B 100, 321 (2010).

3. J. Masson, R. St-Gelais, A. Poulin, and Y.-A. Peter, IEEE J. Quantum Electron. 46, 1313 (2010).

4. M. Fabert, A. Desfarges-Berthelemot, V. Kermne, A. Crunteanu, D. Bouyge, and P. Blondy, Opt. Express 16, 22064 (2008).

5. V. Couderc, A. Crunteanu, M. Fabert, F. Doutre, F. El Bassri, D. Pagnoux, and A. Jalocha, Opt. Express 20, 5524 (2012).

6. A. Crunteanu, D. Bouyge, D. Sabourdy, P. Blondy, V. Couderc, L. Grossard, P. H. Pioger, and A. Barthelemy, J. Opt. A 8, 347 (2006).

7. D. Bouyge, A. Crunteanu, V. Couderc, D. Sabourdy, and P. Blondy, IEEE Photon. Technol. Lett. 20, 991 (2008).

8. M. Fabert, V. Kermne, A. Desfarges-Berthelemot, P. Blondy, and A. Crunteanu, Opt. Lett. 36, 2191 (2011).

9. A. Inoue, T. Komikado, K. Kinoshita, J. Hayashi, and S. Umegaki, Jpn. J. Appl. Phys. 46, L1016-L1018 (2007).

10. W. Lubeigt, J. Gomes, G. Brown, A. Kelly, V. Savitski, D. Uttamchandani, and D. Burns, Opt. Express 19, 2456 (2011).

11. D. Liu, Y. Ning, Y. Zeng, L. Qin, Y. Liu, X. Zhang, L. Zhang, J. Zhang, C. Tong, and L. Wang, Appl. Phys. Express 4, 52104 (2011).

12. MEMSCAP Inc. 12 Alexander Drive, Building 100, Research Triangle Park, NC 27709, USA, www.memscap.com.

13. R. Bauer, G. Brown, and D. Uttamchandani, Micro Nano Lett. 6, 425 (2011).

14. V. Kaajakari, T. Mattila, A. Oja, and H. Seppa, J. Microelectromech. Syst. 13, 715 (2004).

15. J. J. Zayhowski, Opt. Mater. 11, 255 (1999). 